\title{
CONTROL OF ANISOTROPIC ROTOR VIBRATION USING FRACTIONAL ORDER CONTROLLER
}

\author{
Mariusz Czajkowski, Magdalena Gertner \\ Bialystok University of Technology, Department of Automatic Control and Robotics, Bialystok, Poland \\ e-mail:m.czajkowski@doktoranci.pb.edu.pl;m.gertner@doktoranci.pb.edu.pl
}

Monika CiUlKin

Bialystok University of Technology, Department of Mechanics and Applied Computer Science, Bialystok, Poland e-mail: m.ciulkin@doktoranci.pb.edu.pl

\begin{abstract}
This article presents an analysis of a flexible Jeffcott rotor with an active bearing support. The rotor is coupled with lateral and torsional vibrations and a force from the unbalance vector derived from the Lagrangian method. The active support bearing is controlled by FOPID controller. Changing the rotor vibration is followed by shifting rotor angular speed and achieving the natural frequency. Simulation results demonstrate torsional and lateral vibration of thr rotor at the rotating speed near the natural frequency. Consequently, controlling the lateral vibrations mitigates potential damage and improves safety. FOPID controller introduces a new approach to vibration control of a rotating machine.
\end{abstract}

Keywords: rotor dynamic, active bearings, fractional order controller

\section{Introduction}

Application of rotors in modern turbomachinery, particularly in the power generation industry, requires continuous diagnosis and control. Although many studies have been conducted dealing with control of vibration in rotating shafts, there is still no ideal method allowing one to eliminate too high vibration of the machines. Observing rotors vibration during operation with different rotating speeds, allows implementation of various lateral damping on bearings depending on the necessity. Consequently, the control of lateral vibrations mitigates potential damage and improves safety.

Two dynamic systems influence each other when they are coupled. Dynamic lateral and torsional rotor vibrations can be considered separately. This approach is permitted if the effect of coupling is weak (Gosiewski, 2008b; Gosiewski and Muszyńska, 1992; Sawicki et al., 2004).

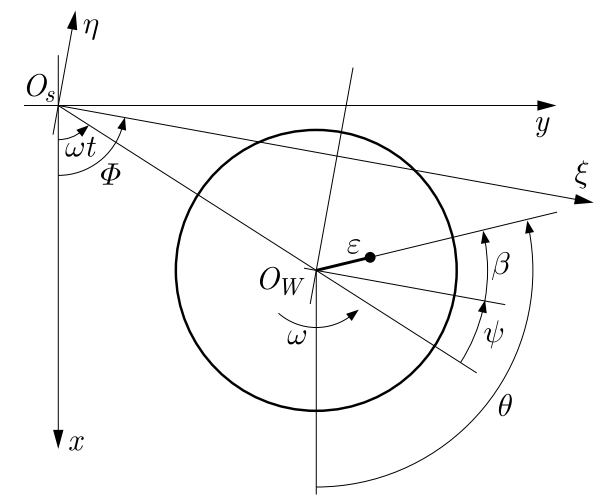

Fig. 1. Section of the rotor in inertial and rotating coordinates 
The system is coupled by the unbalance vector in the model of the rotor (Fig. 1). If the rotor is considered as a weakly damped system it has a range of unstable speeds. The speed ranges are unstable when the poles in diagram of both dynamic systems are crossing each other (Gosiewski and Muszyńska, 1992). This article presents phenomena of vibration in classical dynamical systems (Gosiewski and Muszyńska, 1992) and a relationship for the stability of rotor machinery and vibration control in a closed loop when the rotor speed is in the stable and unstable range. For several decades, control applications in industrial processes have been dominated by the PID controller. Its wide popularity results from the simplicity of design and good performance including low overshoot and small settling time for slow process plants (Astrom and Hagglund, 1995; Biswas et al., 2009). Fast changing process plants require a more sophisticated approach - in several research groups, a fractional order calculus has been proposed (Merrikh-Bayat, 2012). Podlubny (1999) proposed a concept of fractional order controllers and demonstrated the effectiveness of such controllers for actuating the responses of fractional order systems. The transfer function of the fractional order $P I^{\lambda} D^{\mu}$ (FOPID) controller is given by Podlubny (1999) as

$$
G_{c}(s)=K_{P}+K_{I} s^{-\lambda}+K_{D} s^{\mu}
$$

where $K_{P}$ is the proportional gain, $K_{I}$ is the integration gain, $K_{D}$ is the differentiation gain, $\lambda$ is the order of integrator, $\mu$ is the order of differentiator.

\section{Rotor mathematical model}

The mathematical model, called the Jeffcott rotor (Ma et al., 2013), is described as a flexible rotor consisting of a centrally located unbalanced disk attached to a weightless shaft mounted symmetrically on rigid bearings (Gan et al., 2014; Gosiewski, 2008a,b; Gosiewski and Muszyńska, 1992). The stiffness of the shaft is anisotropic (asymmetric) and the damping due to the air resistance effect is assumed to be viscous. The angular position of the unbalanced vector $\varepsilon$ can be used as an indicator and is given by: $\theta(t)=\omega t+\psi(t)+\beta$, where $\omega$ is constant rotating speed of the shaft, $\psi(t)$ is torsional angle, $\beta$ is the angle between the unbalanced vector and the $\xi$ axis. Kinetic and potential energy of the rotor system exhibiting coupling lateral and torsional vibrations can be expressed by the following equations (Sawicki et al., 2004)

$$
T=\frac{m}{2}\left(x_{s}^{2}+y_{s}^{2}\right)+\frac{I_{O}}{2} \dot{\theta} \quad U=\frac{1}{2}\left[\begin{array}{ll}
\xi & \eta
\end{array}\right] \mathbf{K}_{I}\left[\begin{array}{l}
\xi \\
\eta
\end{array}\right]+\frac{K_{T}}{2} \psi^{2}-m g h
$$

where $\mathbf{K}_{I}$ is the shaft stiffness defined by the matrix (Eq. (2.2)) in inertial coordinates for the undisturbed rotor by geometry or influence of the uncracked stiffness $K_{x y}=K_{y x} \equiv 0$. In the case of an unbalanced (anisotropic) rotor, the stiffnesses along the $\xi, \eta$ directions are not equal $\left(K_{x x} \neq K_{y y}\right) . x_{s}=x-\varepsilon \cos (\omega t+\beta)$ and $y_{s}=x+\varepsilon \sin (\omega t+\beta)$ are the coordinates of the centre of mass of the disk in the inertial coordinate system $X Y Z, \xi=x \cos (\omega t)+y \sin (\omega t)$ and $\eta=-x \sin (\omega t)+y \cos (\omega t)$ are the coordinates of the geometric centre of the rotor in the rotating coordinate system $\xi \eta \zeta$ following $\Phi$ with rotating speed

$$
\mathbf{K}_{I}=\left[\begin{array}{ll}
K_{x x} & K_{x y} \\
K_{y x} & K_{y y}
\end{array}\right]
$$

The Rayleigh dissipation function (Lalanne et al., 1998) in lateral vibrations consists of two components. One of these components defines energy dissipation caused by internal dumping, 
the second one describes damping proportional to rotational speed of the rotor in the inertial coordinate system

$$
E_{r}=\frac{1}{2} C_{E}\left(\dot{x}^{2}+\dot{y}^{2}\right)+\frac{1}{2} C_{L}\left(\dot{\xi}^{2}+\dot{\eta}^{2}\right)+C_{T} \psi^{2}
$$

Using the Euler-Lagrange method, the equations of kinetic and potential energy balance (Eq. (2.1)), the Rayleigh dissipation function (Eq. (2.3)) and the non-linear equations of motion for lateral and torsional vibrations of the rotor system (Eqs. (2.4)) are determined

$$
\begin{aligned}
\ddot{x}- & \varepsilon\left[\dot{\theta}^{2} \cos (\theta+\beta)+\ddot{\theta} \sin (\theta+\beta)\right]+\frac{K_{x x}}{m}\left(x \cos ^{2} \theta+y \sin \theta \cos \theta\right) \\
& +\frac{K_{y y}}{m}\left(x \sin ^{2} \theta-y \sin \theta \cos \theta\right)+\frac{C_{E}}{m} \dot{x}+\frac{C_{L}}{m}(\dot{x}+\omega y)=0 \\
\ddot{y}- & \varepsilon\left[\ddot{\theta} \cos (\theta+\beta)-\ddot{\theta}^{2} \sin (\theta+\beta)\right]+\frac{K_{x x}}{m}\left(y \sin ^{2} \theta+x \sin \theta \cos \theta\right) \\
& +\frac{K_{y y}}{m}\left(y \cos ^{2} \theta-x \sin \theta \cos \theta\right)-g+\frac{C_{E}}{m} \dot{y}+\frac{C_{L}}{m}(\dot{y}+\omega x)=0 \\
\ddot{\theta}- & \frac{m \varepsilon}{I_{O}}[\ddot{y} \cos (\theta+\beta)-\ddot{x} \sin (\theta+\beta)]+\frac{m \varepsilon^{2}}{I_{O}} \ddot{\theta}+\frac{m}{I_{O}}\left[\frac{2\left(K_{x x}-K_{y y}\right)}{m}\left(x^{2}-y^{2}\right) \sin 2 \theta\right. \\
& \left.-\frac{K_{x x}-K_{y y}}{m} x y \cos 2 \theta\right]+\frac{K_{T}}{I_{O}}(\theta+\omega t)+\frac{2 K_{T}}{I_{O}}(\dot{\theta}-\omega)=0
\end{aligned}
$$

The next step of modelling is the transition for the non-linear equations of motion in the fixed coordinates to the fixed complex coordinates by specifying the concurrent and backward movement (Eq. (2.5)). Conversion to complex coordinates allows linearisation of the system through coefficients

$$
u=x+\mathrm{j} y \quad \bar{u}=x-\mathrm{j} y
$$

Thus, having the system of equations of motion in the complex inertial coordinates, it can be linearised assuming that the angle of torsion $\theta=\omega t+\psi$ is close to the angle of rotation $\Phi=\omega t$, where $\psi$ is a relatively small angular displacement describing torsional vibration of the shaft. A linearised Taylor series (Eqs. (2.6)) is used. In this step, the first expressions of decomposition of the exponential function in Eq. $(2.6)_{1}$ is taken. The non-linear elements have been skipped (Gosiewski and Muszyńska, 1992). As a result of this procedure, we obtain linearised equations of motion in the complex inertial coordinates, and using formulas (Eqs. $(2.6)_{2-5}$ ), equations in the real rotating coordinates are obtained

$$
\begin{aligned}
& \mathrm{e}^{ \pm \mathrm{j} \psi}=1 \pm \mathrm{j} \psi+\ldots \quad \quad u=w \mathrm{e}^{\mathrm{j} \omega t} \quad \bar{u}=\bar{w} \mathrm{e}^{-\mathrm{j} \omega t} \\
& w=\xi+\mathrm{j} \eta \quad \bar{w}=\xi-\mathrm{j} \eta
\end{aligned}
$$

Then the rotor model is determined by

$$
\begin{aligned}
\ddot{\xi}= & 2 \omega \dot{\eta}+\omega^{2} \xi-\frac{C_{E}}{m}(\dot{\xi}-\omega \eta)-\frac{C_{L}}{m} \dot{\xi}-\frac{K_{x x}}{m} \xi-\varepsilon \omega^{2} \psi \sin \beta+2 \varepsilon \omega \dot{\psi} \cos \beta \\
& +\varepsilon \ddot{\psi} \sin \beta+\varepsilon \omega^{2} \cos \beta+g \sin (\omega t) \\
\ddot{\eta}= & -2 \omega \dot{\xi}+\omega^{2} \eta-\frac{C_{E}}{m}(\dot{\eta}-\omega \xi)-\frac{C_{L}}{m} \dot{\eta}-\frac{K_{y y}}{m} \eta-\varepsilon \omega^{2} \psi \cos \beta+2 \varepsilon \omega \dot{\psi} \sin \beta \\
& +\varepsilon \ddot{\psi} \cos \beta+\varepsilon \omega^{2} \sin \beta+g \cos (\omega t) \\
\ddot{\psi}= & -\frac{C_{T}}{I_{O}}-\frac{m \varepsilon^{2}}{I_{O}} \omega^{2} \psi-\frac{K_{T}}{I_{O}} \psi-\frac{\varepsilon}{I_{O}} K_{y y} \xi \sin \beta+\frac{\varepsilon}{I_{O}} K_{x x} \eta \cos \beta-\frac{m \varepsilon}{I_{O}} g \cos (\omega t+\beta)
\end{aligned}
$$


The coefficients of the rotor dynamic equation obtained in this way are time dependent, which means that vibration of the rotor can be considered as parametric vibration. It is known that vibrations of machines are unstable in a range of parameters. One of the rotor parameter is the angular speed, which is changing while working. For the purpose of solving the eigenvalue problem, free lateral and torsional vibration of the rotor should be determined. Free vibrations are deprived of external excitations and are defined by the following equations

$$
\begin{aligned}
\ddot{\xi}= & 2 \omega \dot{\eta}+\omega^{2} \xi-\frac{C_{E}}{m}(\dot{\xi}-\omega \eta)-\frac{C_{L}}{m} \dot{\xi}-\frac{K_{x x}}{m} \xi-\varepsilon \omega^{2} \psi \sin \beta \\
& +2 \varepsilon \omega \dot{\psi} \cos \beta+\varepsilon \ddot{\psi} \sin \beta \\
\ddot{\eta}= & -2 \omega \dot{\xi}+\omega^{2} \eta-\frac{C_{E}}{m}(\dot{\eta}-\omega \xi)-\frac{C_{L}}{m} \dot{\eta}-\frac{K_{y y}}{m} \eta-\varepsilon \omega^{2} \psi \cos \beta \\
& +2 \varepsilon \omega \dot{\psi} \sin \beta+\varepsilon \ddot{\psi} \cos \beta \\
\ddot{\psi}= & -\frac{C_{T}}{I_{O}}-\frac{m \varepsilon^{2}}{I_{O}} \omega^{2} \psi-\frac{K_{T}}{I_{O}} \psi-\frac{\varepsilon}{I_{O}} K_{y y} \xi \sin \beta+\frac{\varepsilon}{I_{O}} K_{x x} \eta \cos \beta
\end{aligned}
$$

Using the Laplace transformation, differential equations (2.8) take the form of coupled equations with the complex variable $s=-\mathrm{j} \omega$

$$
\left[\begin{array}{ccc}
A_{1 d}(s) & -B_{d}(s) & -D(s) \\
B_{d}(s) & A_{2 d}(s) & -F(s) \\
-H(s) & -K(s) & C(s)
\end{array}\right]\left[\begin{array}{l}
\xi \\
\eta \\
\psi
\end{array}\right]=\left[\begin{array}{l}
0 \\
0 \\
0
\end{array}\right]
$$

where

$$
\begin{array}{ll}
A_{1 d}(s)=s^{2}+\frac{1}{m}\left(C_{E}+C_{L}\right) s+\frac{K_{y y}}{m}-\omega^{2} & A_{2 d}(s)=s^{2}+\frac{1}{m}\left(C_{E}+C_{L}\right) s+\frac{K_{x x}}{m}-\omega^{2} \\
B_{d}(s)=2 \omega s+\frac{C_{E}}{m} \omega & C(s)=s^{2}+\frac{C_{T}}{I_{O}} s+\frac{m \varepsilon^{2}}{I_{O}} \omega^{2}+\frac{K_{T}}{I_{O}} \\
D(s)=\varepsilon \sin \beta s^{2}+2 \varepsilon \omega \cos \beta s-\varepsilon \omega^{2} \cos \beta & F(s)=\varepsilon \cos \beta s^{2}+2 \varepsilon \omega \sin \beta s-\varepsilon \omega^{2} \cos \beta \\
H(s)=K_{y y} \frac{\varepsilon}{I_{O}} \sin \beta & K(s)=K_{x x} \frac{\varepsilon}{I_{O}} \cos \beta
\end{array}
$$

The determinant of the main matrix in equation (Eq. (2.9)) is the characteristic polynomial of the rotor model. Comparing this characteristic polynomial to zero, poles of the system are obtained.

Using such a characteristic equation, stability of the system can be determined. When the system is stable, all elements (poles) of the characteristic equation have negative real parts. Checking the roots of the characteristic equation in the rotational speed of the rotor $\omega$ characteristic shows that the speed of the rotor is stable. The rotor parameters are chosen to correspond to real machines (Sawicki et al., 2004). The map in Fig. 2 shows the course of the poles depending on the rotating speed. Unstable rotating speed occurs when the poles change the sign in the real part. Furthermore, unstable rotating speed occurs when the imaginary part of the trajectories intersect (Gosiewski, 2008a,b). The first area corresponds to the natural frequency $\omega_{1} \cong\left(\sqrt{K_{x x} / m}+\sqrt{K_{y y} / m}\right) / 2$, the second $\omega_{2} \cong \sqrt{K_{T} / I_{O}}-\left(\sqrt{K_{x x} / m}+\sqrt{K_{y y} / m}\right) / 2$ and the third $\omega_{3} \cong \sqrt{K_{T} / I_{O}}+\left(\sqrt{K_{x x} / m}+\sqrt{K_{y y} / m}\right) / 2$.

Real parameters listed in Table 1 are chosen for the model in order to emphasize the importance of examining real life applications. 

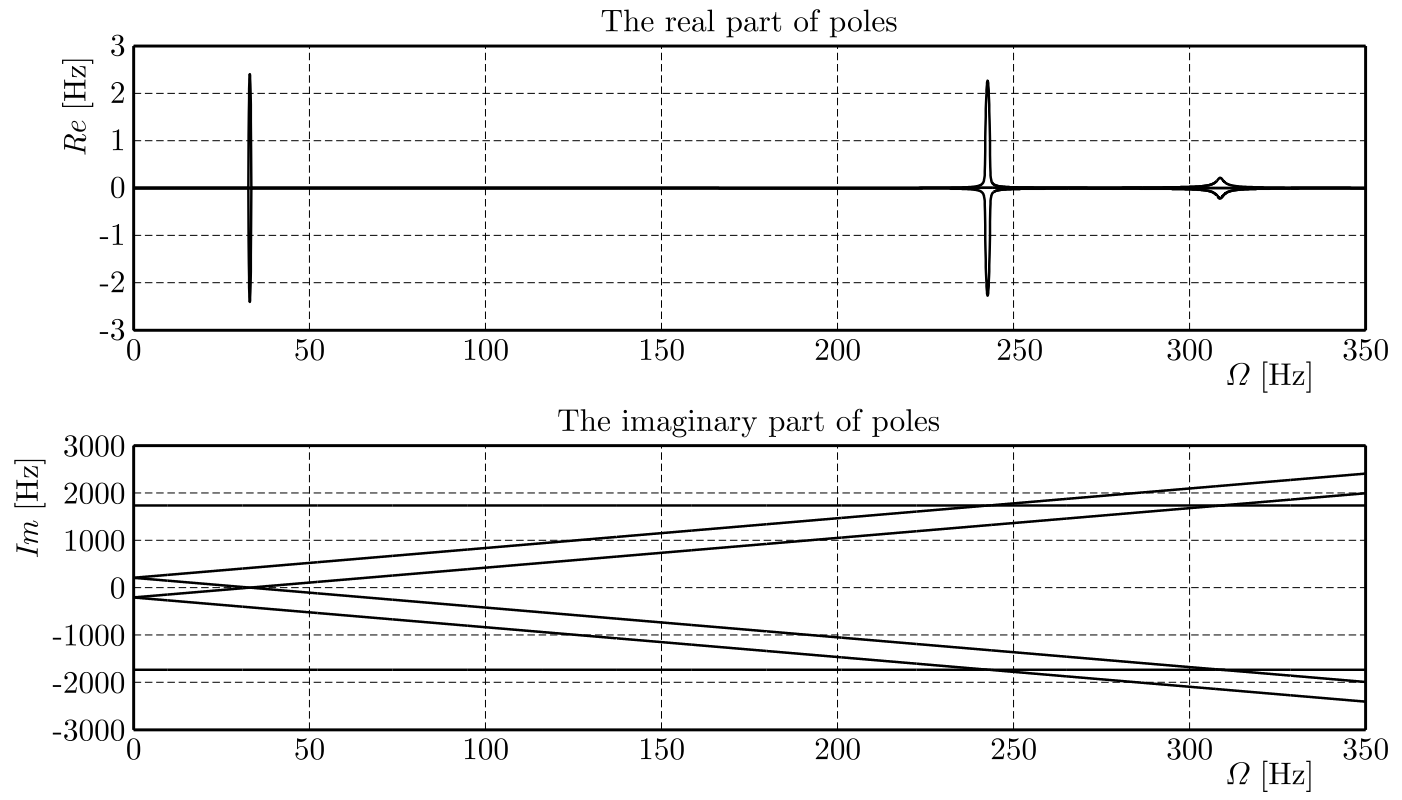

Fig. 2. Distribution poles in the rotation frequency

Table 1. Simulation parameters (Sawicki et al., 2004)

\begin{tabular}{|l|c|c|}
\hline \multicolumn{1}{|c|}{ Physical parameters } & Value & Units \\
\hline \hline Disk mass $m$ & 20 & $\mathrm{~kg}$ \\
\hline Disk polar moment of inertia $I_{O}$ & 0.3 & $\mathrm{~kg} / \mathrm{m}^{2}$ \\
\hline Shaft stiffness $K_{x x}$ & $8.82 \cdot 10^{5}$ & $\mathrm{~N} / \mathrm{m}$ \\
\hline Shaft stiffness $K_{y y}$ & $8.42 \cdot 10^{5}$ & $\mathrm{~N} / \mathrm{m}$ \\
\hline Torsional shaft stiffness $K_{T}$ & $9.00 \cdot 10^{5}$ & $\mathrm{~N} / \mathrm{m}$ \\
\hline Range of stiffness $\Delta K$ & $10 \%$ & \\
\hline Unbalance eccentricity $\varepsilon$ & $1.5 \cdot 10^{-3}$ & $\mathrm{~m}$ \\
\hline Unbalance phase angle $\beta$ & 30 & degrees \\
\hline Lateral damping ratio $C_{L}$ & 0.01 & - \\
\hline External damping ratio $C_{E}$ & 0.01 & - \\
\hline Torsional damping ratio $C_{T}$ & 0.001 & - \\
\hline
\end{tabular}

\section{Realization of the fractional order controller}

There are many methods of finding integer or discrete transfer functions which approximate a fractional derivative. For example, Merrikh-Bayat (2011) approximated the transfer function of controller with an integer order transfer function. Valsa and Brancik (1998) used the inverse Laplace transformations, and more methods can be found in Vinagre et al. (2000)

The Oustaloup Recursive Approximation (ORA) is widely used in finding a rational integer-order approximation for fractional-order integrators and differentiators of the form $s^{r}$, where $r \in(-1,1)$ and is defined by Eq. (3.1) (Merrikh, 2012; Oustaloup et al., 2000; Vinagre et al., 2000)

$$
s^{r} \approx k \prod_{n=1}^{N} \frac{1+\frac{s}{\omega_{z, n}}}{1+\frac{s}{\omega_{p, n}}}
$$


where Eq. (3.1) is determined by Eqs. (3.2)

$$
\begin{aligned}
& \omega_{z, 1}=\omega_{l} \sqrt{\eta} \quad \omega_{p, n}=\omega_{z, n} \alpha \quad n=1, \ldots, N \\
& \omega_{z, n+1}=\omega_{p, n} \eta, \quad n=1, \ldots, N-1 \quad \alpha=\left(\frac{\omega_{h}}{\omega_{l}}\right)^{\frac{r}{N}} \quad \eta=\left(\frac{\omega_{h}}{\omega_{l}}\right)^{\frac{1-r}{N}}
\end{aligned}
$$

where $N$ is the number of poles and zeros, $\omega_{h}$ is high transitional frequency, $\omega_{l}$ is low frequency, $\omega_{u}$ is the unit gain frequency and the central values of a band of frequencies geometrically distributed around it and defined by $\omega_{u}=\sqrt{\omega_{l} \omega_{h}}$. The Oustaloup-Recursive-Approximation for a fractional order differentiator enable one to obtain the transfer function of an integral element of the order of $1 / 2$ (Xue et al., 2001). The following variables should be given: $r$ - fractional order as in $s^{r}$, where $r$ is a real number, $N$ - order of the finite transfer function approximation for both (num/den), $\omega_{l}$ - low frequency limit of the range of frequency of interest, $\omega_{h}$ - upper frequency limit of the range of frequency of interest. The frequencies $\omega_{l}$ and $\omega_{h}$ should be selected before approximation of the integer element of the fractional $P I^{\lambda} D$ controller. If the frequency of the input signal is in a different range than $\omega_{l}$ and $\omega_{h}$, the fractional $P I^{\lambda} D$ controller will not be able to work properly - the integer part of the controller will not act like a integer.

In further simulation, $\lambda=1 / 2, \omega_{l}=3 \cdot 10^{4} \mathrm{~Hz}, \omega_{h}=2 \cdot 10^{-4} \mathrm{~Hz}$ and $N=1,5,11$ are considered.

Figure 3 shows a comparison of a fractional order with the classical order in the Bode characteristic. As it can be seen, the 11-th approximation of the integrator element gives a sufficient result for the factor in the integrator description and gives similar results for the phase characteristic to the classical order. According to the range of frequencies, the systems have different quality of control. If the order of approximation increases, the ripple of phases decreases.

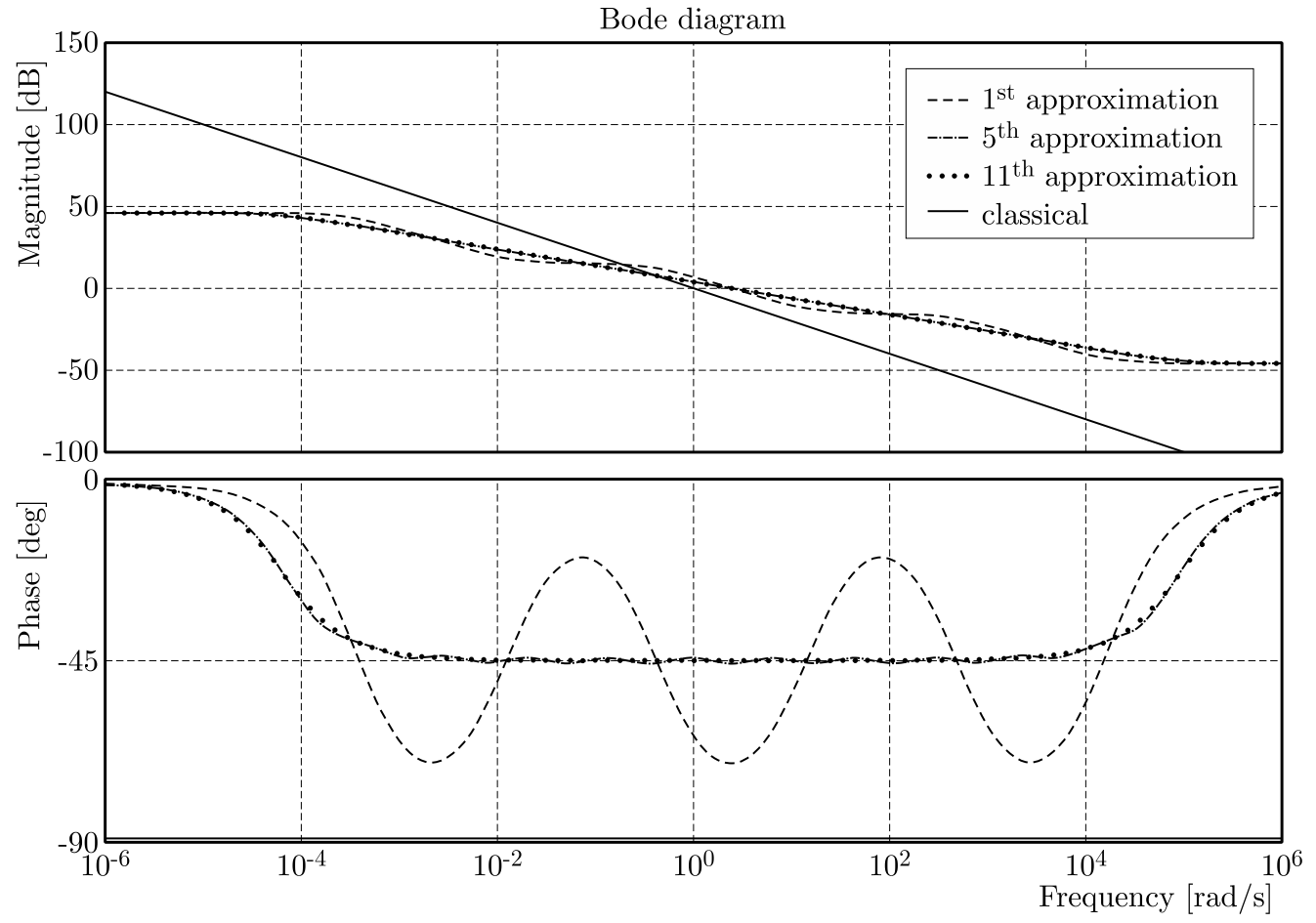

Fig. 3. Comparison of the fractional order with the classical order in the Bode characteristic

In Fig. 4, a comparison of the poles-zeros plot of different approximations of the fractional integer with the classical integer are shown. From this characteristic, several numbers of poles and zeros of the systems have been received. All systems are within stability boundary and do 
not have imaginary parts. With increasing approximation, the zeros and poles move closer to the area of stability.

Poles-zeros map of $1^{\text {st }}$ approximation of $I^{\lambda}$

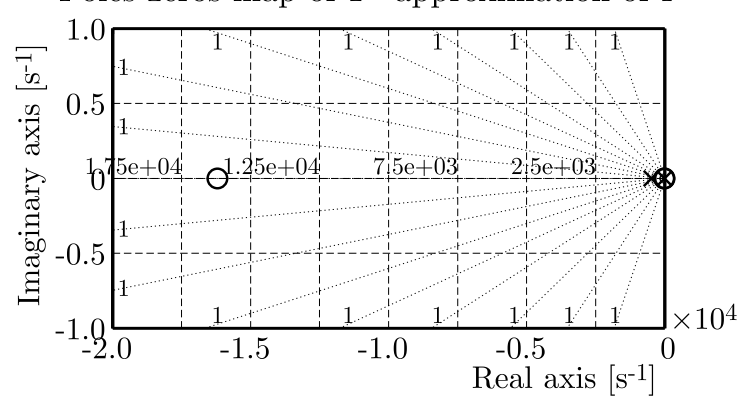

Poles-zeros map of $11^{\text {st }}$ approximation of $I^{\lambda}$

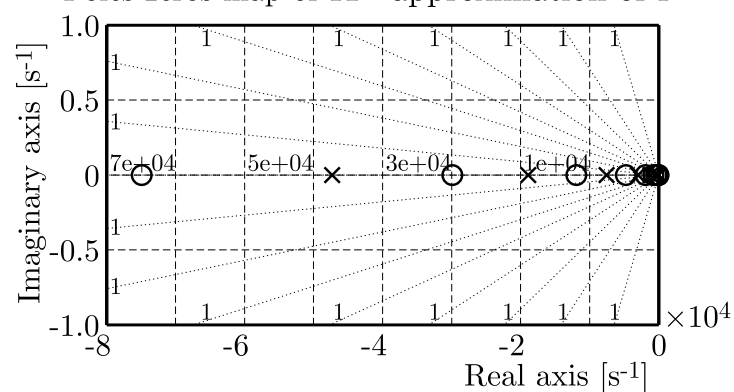

Poles-zeros map of $5^{\text {st }}$ approximation of $I^{\lambda}$

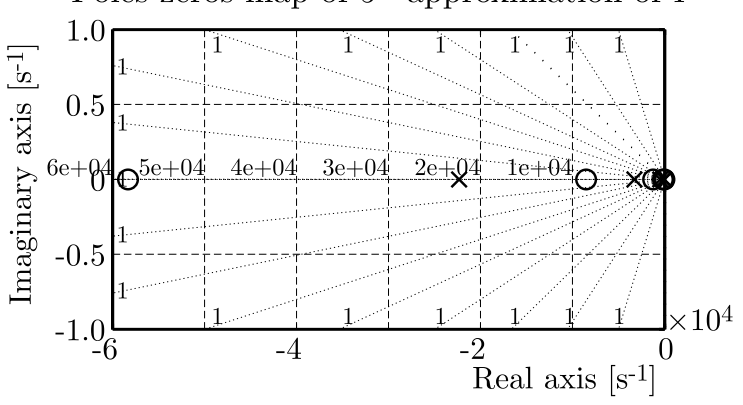

Poles-zeros map of classical $I$

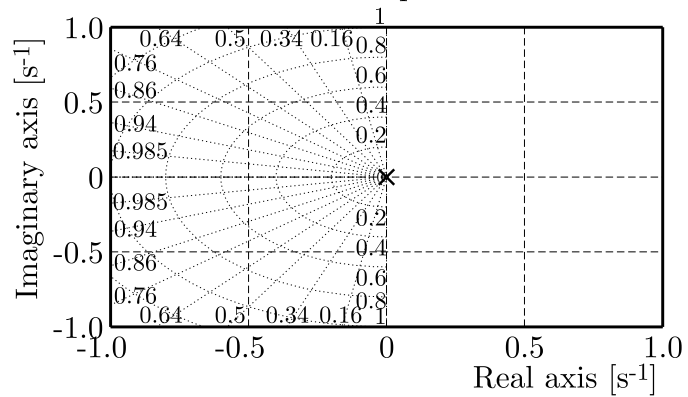

Fig. 4. Comparison of the fractional order with the classical order in poles-zeros map

Figure 5 shows the step response of a different number of approximations of the integrator used in the fractional order controller. The comparison shows that the system with a lower number of approximations achieves faster the set point.

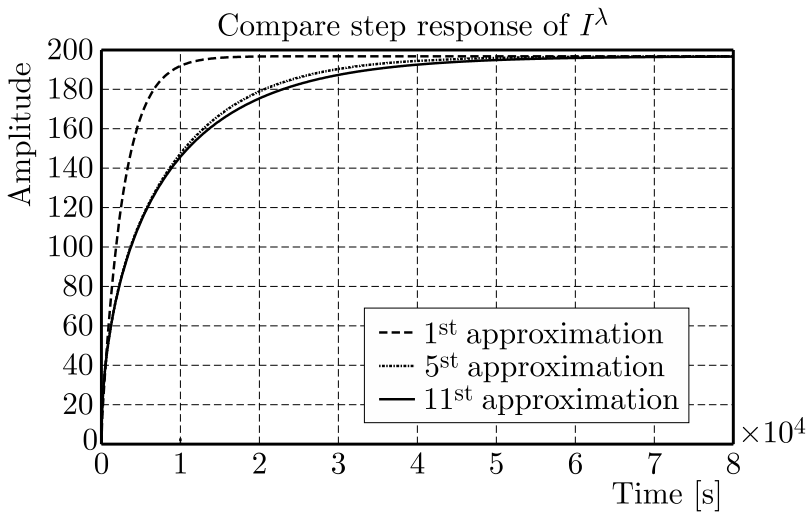

Fig. 5. Comparison of the fractional order with the classical order on the step response

In the low range of frequencies the integral element behave like a proportional element, in the higher range like an object with damped signals. This leads to non-linear action of the integral element which can precipitate internal signal saturation for long prevalence of a fixed offset value and "low pass filter" in the case of a large dynamic offset.

\section{Open-loop and closed-loop system}

Free vibration at a stable speed $(30 \mathrm{~Hz})$, close to the first unstable speed, is shown in Fig. 6 . Vibration along the axis $\eta$ and $\xi$ have similar amplitudes and are shifted in phase by $\pi / 2 \mathrm{rad}$ in 
which the controller can work with information about the value $\xi$. Vibration of $\psi$ depends on $\eta$ and $\xi$, so if $\eta$ and $\xi$ are minimized then, vibration of $\psi$ is also minimized.
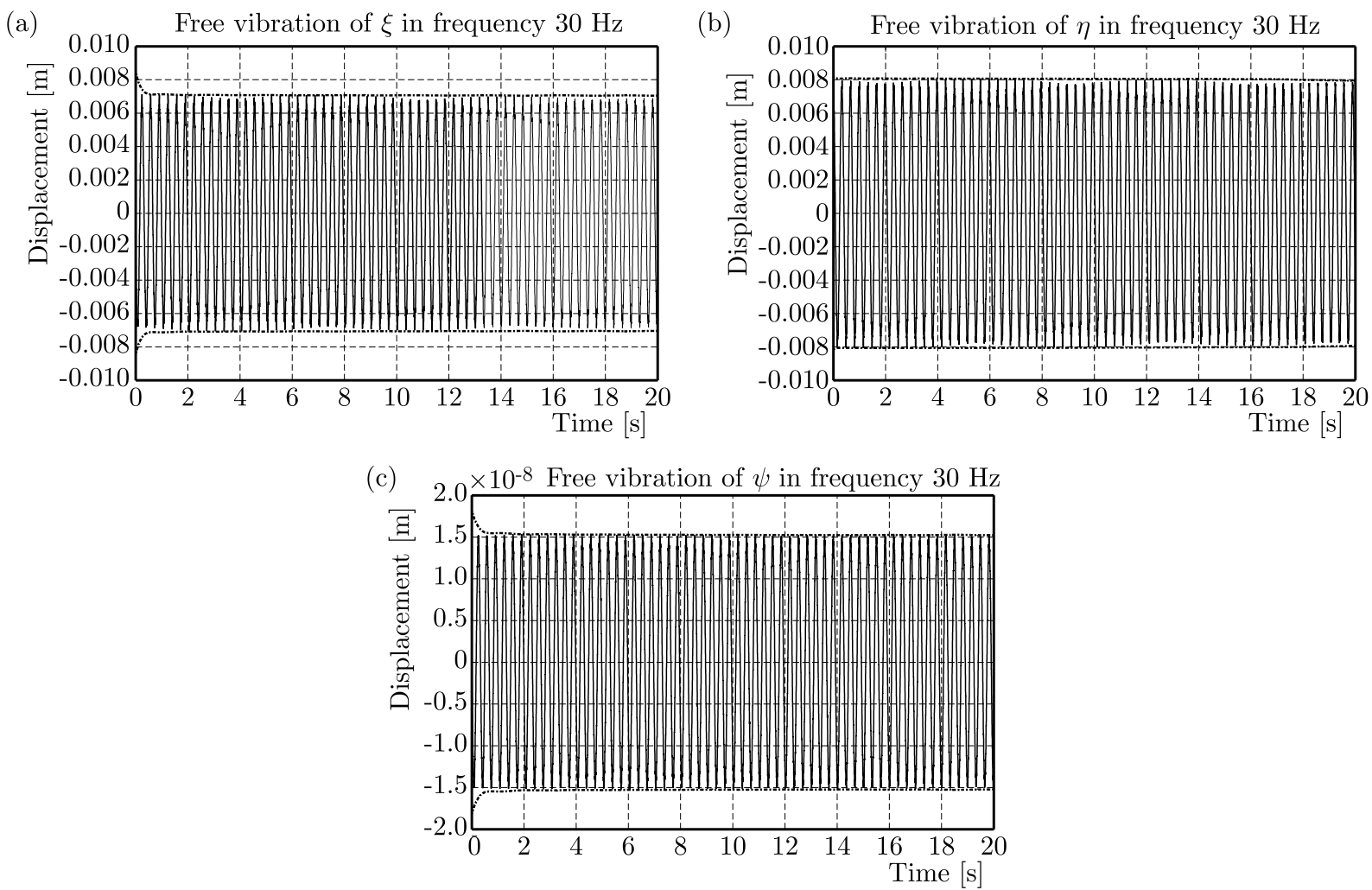

Fig. 6. Free vibration of the rotor, (a) vibration of $\xi$, (b) vibration of $\eta$, (c) vibration of $\psi$

The classical PID controller and fractional $P I^{\lambda} D$ controller have been designed for stable rotation speed $\omega=30 \mathrm{~Hz}$. The rotating speed $\omega=30 \mathrm{~Hz}$ has been selected as a stable speed by the characteristic shown in Fig. 2. This vibrations have the amplitude in constant value (not increasing). Vibrations in the $\xi, \eta$ axes have similar amplitudes and differ by a phase shift $\pi / 2 \mathrm{rad}$ from each other. The controller measuring the amplitude of vibration in the $\xi$ axis does not need the information in the $\eta$ axis because of the similarity. Vibrations in the $\psi$ axis are coupled with vibrations in the $\xi$ and $\eta$ axes by the stiffness matrix (Eq. (2.4)). Minimising the vibrations in the $\xi$ and $\eta$ vertical axis decreases the vibrations of the $\xi$ and $\eta$ directions.

The concept of realization of the rotor vibration controller consists in measuring the amplitude of vibration in the $\xi$ axis and varying the stiffness of the active supports within the range $\Delta K$ (Fig. 7). The controller changes the transverse stiffness of the system by adding to the initial stiffness $K_{x x}$, and $K_{y y}$ by adding the processing stiffness $\Delta K$. The final value of stiffness is $\Delta K_{x x}=\Delta K_{y y}=K_{x x}+\Delta K=K_{y y}+\Delta K$ (Fig. 7).

The controller settings for the rotating speed $\omega=30 \mathrm{~Hz}$ are shown in Table 2. The first case is tuned for the $P I^{\lambda} D$ controller, the second one for a classical PID controller. As the two elements of the controller do not differ in structure and the integral component $I$ acts similarly in both cases, the responses of the controls have been tested for the same two settings.

Simulations have been carried for two stable rotating speeds $(30 \mathrm{~Hz}, 36 \mathrm{~Hz})$ and one unstable rotating speed $(32 \mathrm{~Hz})$. The results are shown in Figs. 8-10 and in Table 3.

Simulations of rotor vibrations have been carried out at a constant rotating speed. Simulations present only vibration in the $\xi$ axis. All results of the simulstion are presented in Table 3 . To present stable vibrations of the rotor, the operation of the controller has been started at the second second of simulation. In the first simulation at the rotating speed $\omega=30 \mathrm{~Hz}$ the con- 


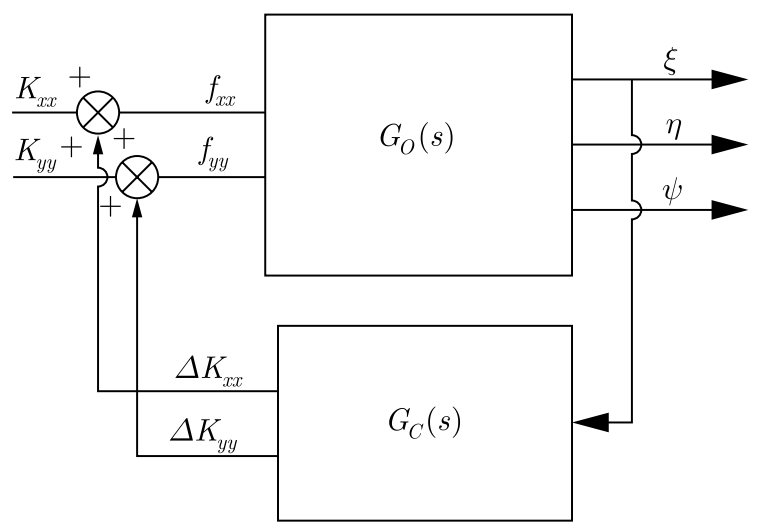

Fig. 7. Close loop system

Table 2. Controller settings

\begin{tabular}{|c|c|c|c|}
\hline Settings & First case & Second case & Units \\
\hline \hline$k_{p}$ & $1.4139 \cdot 10^{3}$ & $2.5194 \cdot 10^{3}$ & - \\
\hline$T_{i}$ & 14.9496 & 7.8666 & $s$ \\
\hline$k_{d}$ & 8 & 8 & - \\
\hline$T_{d}$ & 1.69787 & 3.8445 & $s$ \\
\hline
\end{tabular}
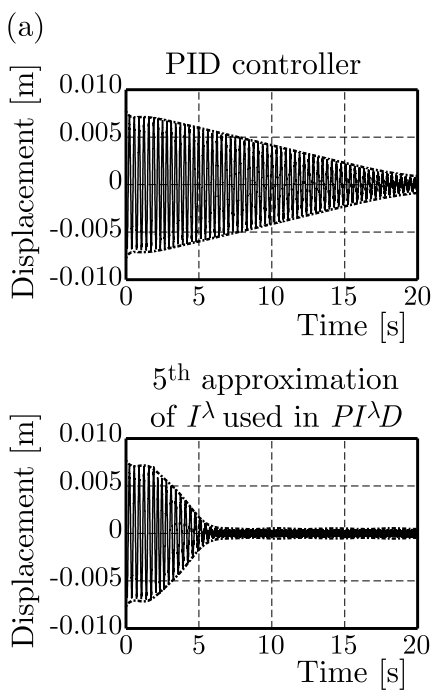

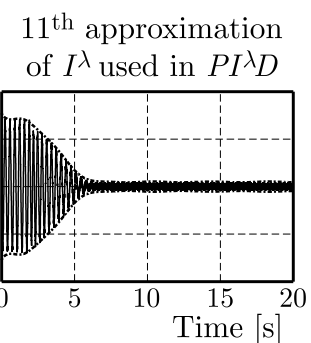

$1^{\text {th }}$ approximation

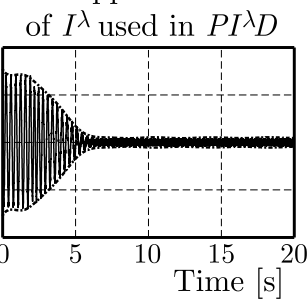

(b)

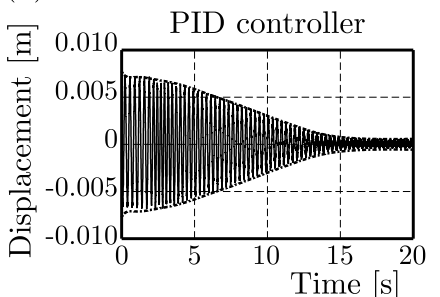

$5^{\text {th }}$ approximation

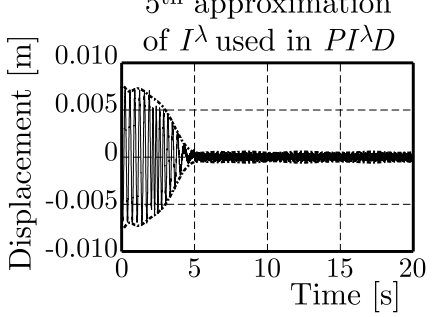

$11^{\text {th }}$ approximation of $I^{\lambda}$ used in $P I^{\lambda} D$

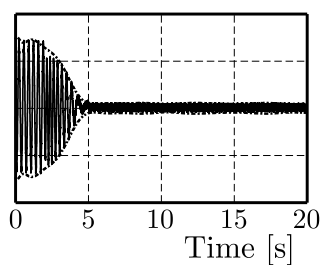

$1^{\text {th }}$ approximation

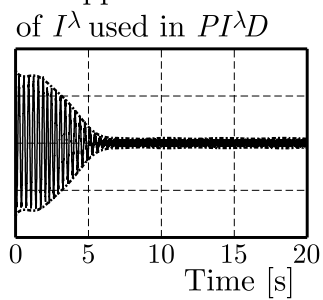

Fig. 8. Comparison of controllers at the rotating speed $30 \mathrm{~Hz}$. Vibration in the $\xi$ direction with controllers with first settings (a) and with second settings (b)

trollers have first settings (Table 2). The settling time in the system with the fractional order controller is shorter than in the classical PID controller (Fig. 9). The response of the system with $P I^{\lambda} D$ controllers for all order approximations has close settling time. The amplitude of settling vibrations in the system with FOPID controllers is lower than in the system with the classical PID controller.

In the next simulation, the second set of settings of the controllers (Table 2) with the same rotor rotating speed $(\omega=30 \mathrm{~Hz})$ has been used. The FOPID controllers are still better than the classical PID controller. The systems with the FOPID controllers have a shorter settling time and lower amplitudes of vibrations.

Next simulations present control vibration at an unstable rotating speed ( $\omega=32 \mathrm{~Hz})$ (Fig. 9). 

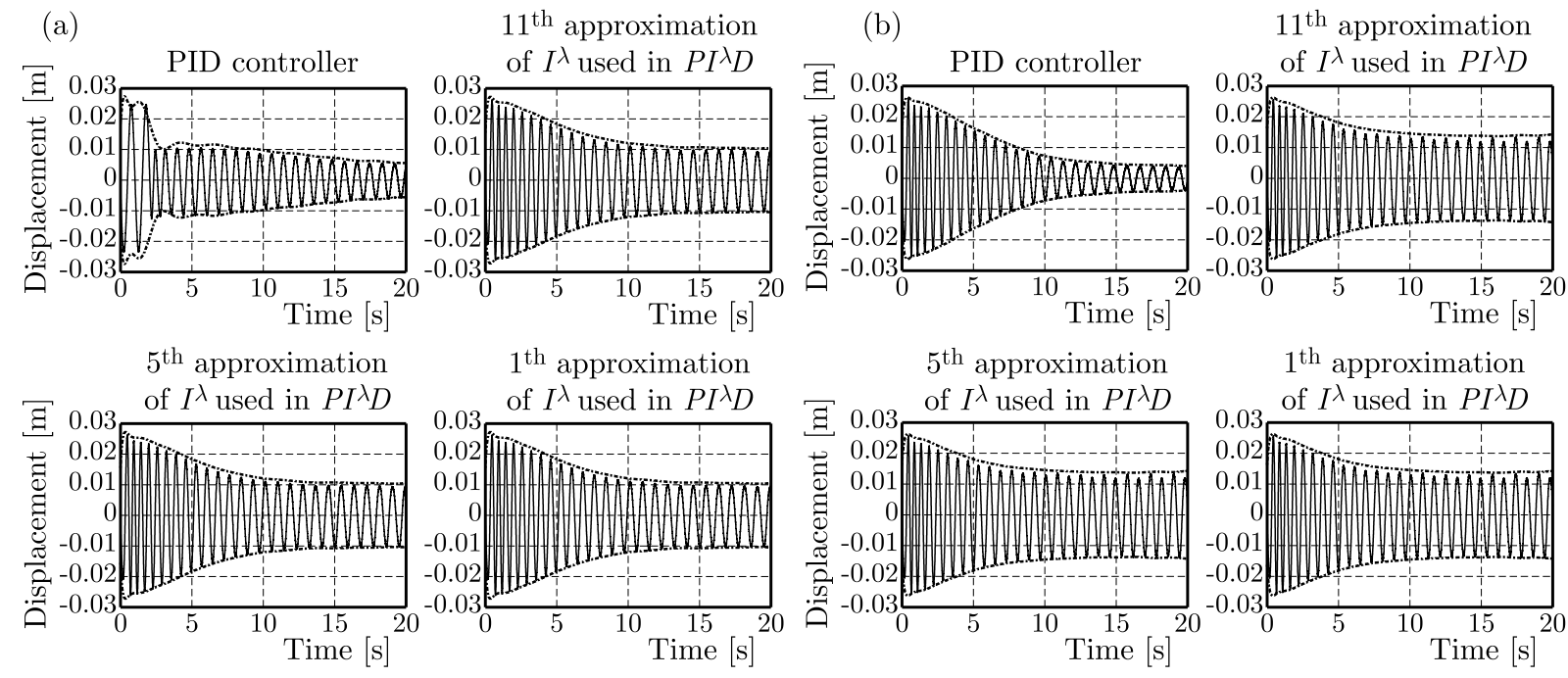

Fig. 9. Comparison of controllers at the rotating speed $32 \mathrm{~Hz}$. Vibration in the $\xi$ direction with controllers with first settings (a) and with second settings (b)
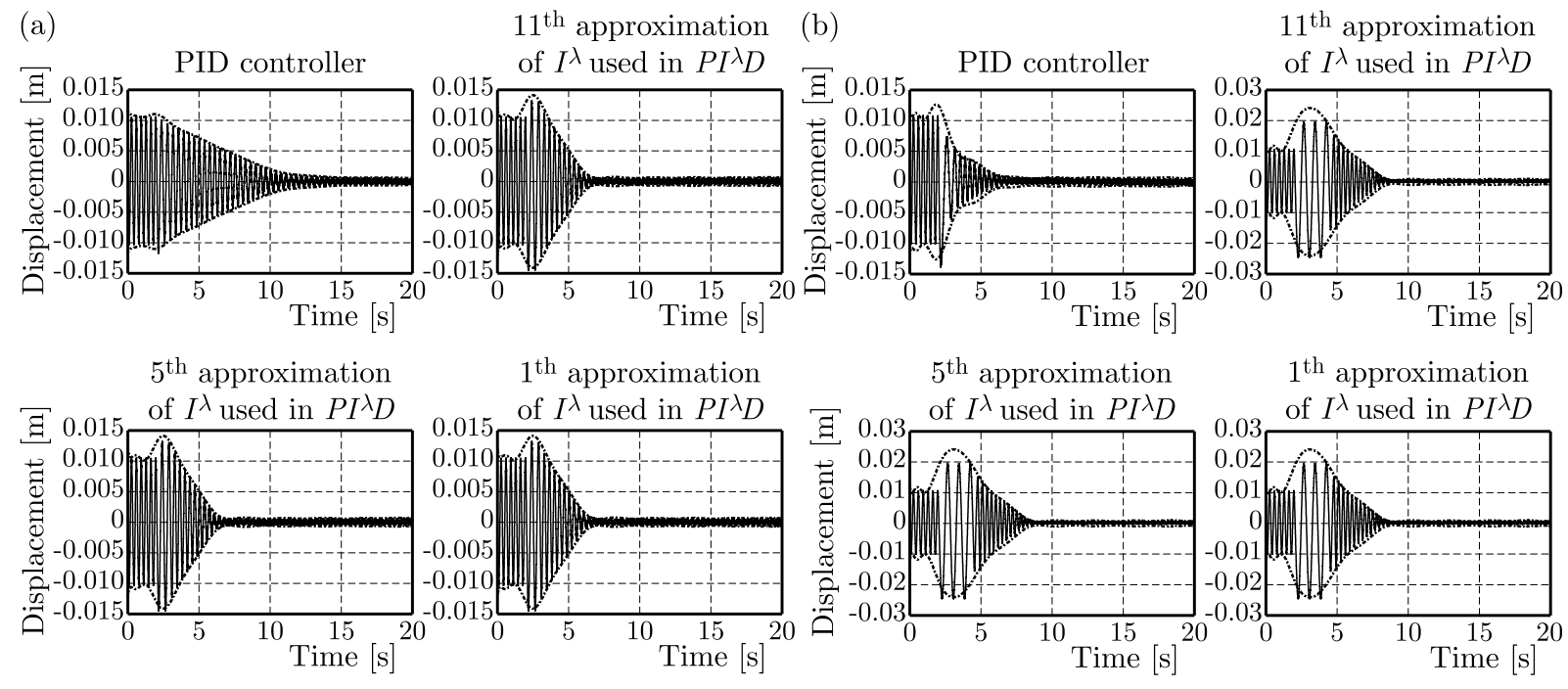

Fig. 10. Comparison of controllers at the rotating speed $36 \mathrm{~Hz}$. Vibration in the $\xi$ direction with controllers with first settings (a) and with second settings (b)

Because the amplitude of vibration goes to infinity, the controllers have been started at the beginning of simulation. The systems with the classical PID controllers are characterized by a better stablisation process. Simulations for systems with different order of approximations of $P I^{\lambda} D$ present various amplitudes for settling vibrations, but the settling times are constant. With an increase in the order of the approximation, the amplitudes of settling vibrations decrease for all settings of the controller. The systems with classical PID controllers have longer settling times but achieve a lower amplitude of settling vibrations.

In the next part of the numerical research, the response of the close loop systems with controllers have been tested at a stable speed $36 \mathrm{~Hz}$ (Fig. 10). This is intended to test how the settings and types of the controllers are resistant to a change in the rotating speed. In both cases, overshoot is observable, but in the classical controller the overshoot has a smaller value than in the fractional order controller. The vibrations in steady states of the system with the fractional order controller reach smaller values. 
Table 3. Comparison of the simulations results

\begin{tabular}{|c|c|c|c|c|c|}
\hline Controller & Settings & $\begin{array}{c}\text { Order of } \\
\text { of approximation }\end{array}$ & $\begin{array}{c}\text { Rotating } \\
\text { speed }[\mathrm{Hz}]\end{array}$ & $\begin{array}{l}\text { Settling } \\
\text { time }[\mathrm{s}]\end{array}$ & $\begin{array}{c}\text { Settling } \\
\text { vibration }[\mathrm{m}]\end{array}$ \\
\hline \multirow{6}{*}{$P I D$} & \multirow[t]{3}{*}{ First case } & & 30 & 18 & $9 \cdot 10^{-4}$ \\
\hline & & & 32 & 19 & $5.6 \cdot 10^{-3}$ \\
\hline & & & 36 & 10 & $6.4 \cdot 10^{-4}$ \\
\hline & \multirow[t]{3}{*}{ Second case } & & 30 & 12 & $5.6 \cdot 10^{-4}$ \\
\hline & & & 32 & 14 & $4.1 \cdot 10^{-3}$ \\
\hline & & & 36 & 4 & $6.1 \cdot 10^{-4}$ \\
\hline \multirow{9}{*}{$P I^{\lambda} D$} & \multirow[t]{4}{*}{ First case } & 1-st, 5-th, 11-th & 30 & 4 & $4.6 \cdot 10^{-4}$ \\
\hline & & 1-st, 5-th & 32 & 12 & $1.06 \cdot 10^{-2}$ \\
\hline & & 11-th & 32 & 12 & $1 \cdot 10^{-2}$ \\
\hline & & 1-st, 5-th, 11-th & 36 & 4.5 & $6.8 \cdot 10^{-4}$ \\
\hline & \multirow[t]{5}{*}{ Second case } & 1-st, 5-th, 11-th & 30 & 3 & $5.2 \cdot 10^{-4}$ \\
\hline & & 1 -st & 32 & 10 & $1.39 \cdot 10^{-2}$ \\
\hline & & 5 -th & 32 & 10 & $1.37 \cdot 10^{-2}$ \\
\hline & & 11-th & 32 & 10 & $1.3 \cdot 10^{-2}$ \\
\hline & & 1-st, 5-th, 11-th & 36 & 6.5 & $6.7 \cdot 10^{-4}$ \\
\hline
\end{tabular}

\section{Conclusion}

Taking into account the coupling between torsional and bending vibrations for the Jeffcott model, an analytical solution has been developed. The inclusion of Eq. (2.7) $)_{3}$ for torsional vibration is used to achieve this result. Numerical simulations of the anisotropic rotor are carried out by applying an unbalance excitation, gravity forces and a torsional excitation. The torsional and lateral vibrations and the settling time are compared by using a different approach to control theory. The classical controller and the fractional order controller are used in the closed loop system. The investigations have been carried out on the effects of the type of controllers on rotor vibration and revealed that PID controllers are more efficient around the point in which they are tuned and have lower resistance to changes in the speed of rotation. For unstable vibrations, better results give systems with the classical PID controller. The authors concluded that a good approach to controlling vibration of the rotor at a stable speed is to build a system with switching parameters of the FOPID controller relative to the rotational speed. A real life problem may be still the proper choice of actuators corresponding to the reaction to changes in the stiffness control signal of the rotor and, more specifically, in its bearing. However, the proposed concept of a new method of control for an anisotropic rotor using a fractional order controller requires experimental confirmation, which is underway.

\section{Acknowledgements}

The authors acknowledge the financial support by Faculty of Mechanical Engineering, Bialystok University of Technology (MB/WM/7/2014).

\section{References}

1. Astrom K., Hagglund T., 1995, PID Controllers: Theory, Design and Tuning, Instrument Society of America

2. Biswas A., Das S., Abraham A., Dasgupta S., 2009, Design of fractional-order $P I^{\lambda} D^{\mu}$ controllers with an improved differential evolution, Engineering Applications of Artificial Intelligence, 22, 343-350 
3. Gan C.B., Wang Y.H., Yang S.X., CaO Y.L., 2014, Nonparametric modeling and vibration analysis of uncertain Jeffcott rotor with disc offset, International Journal of Mechanical Sciences, 78, $126-134$

4. Gosiewski Z., 2008a, Analysis of coupling mechanism in lateral/torsional rotor vibrations, Journal of Theoretical and Applied Mechanics, 46, 4, 829-844

5. Gosiewski Z., 2008b, Control-oriented modelling and control of rotor vibration, Acta Mechanica et Automatica, 2, 2, 21-38

6. Gosiewski Z., Muszyńska A., 1992, Dynamics of Rotating Machinery (in Polish), Wydaw. Wyższej Szkoły Inżynierskiej, Koszalin

7. Lalanne M., Ferraris G., 1998, Rotordynamics Prediction in Engineering, John Wiley \& Sons Ltd.

8. Ma H., Li H., Niu H., Song R, Wen B., 2013, Nonlinear dynamic analysis of a rotor-bearing-seal system under two loading conditions, Journal of Sound and Vibration, 332, 6128-6154

9. Merrikh-Bayat F., 2011, Efficient method for time-domain simulation of the linear feedback systems containing fractional order controllers, ISA Transactions, 50, 2, 170-176

10. Merrikh-Bayat F., 2012, Rules for selecting the parameters of Oustaloup recursive approximation for the simulation of linear feedback systems containing $P I^{\lambda} D^{\mu}$ controller, Communications in Nonlinear Science and Numerical Simulation, 17, 1852-1860

11. Oustaloup A., Levron F., Mathieu B., Nanot F., 2000, Frequency-band complex noninteger differentiator: characterization and synthesis, IEEE Transactions on Circuits and Systems. I: Fundamental Theory and Applications, 47, 1, 25-39

12. Podlubny I., 1999, Fractional-order systems and $P I^{\lambda} D^{\mu}$-controllers, IEEE Transactins on Automatic Control, 44, 1

13. Sawicki J.T., BaAklini G.Y., Gyekenyesi A.L., 2004, Dynamic analysis of accelerating cracked flexible rotor, Turbo ASME Turbo Expo Conference

14. VAlsa J., BranciK L., 1998, Approximate formulae for numerical inversion of Laplace transforms, International Journal of Numerical Modelling: Electronic Networks, Devices and Fields, 11, 153-166

15. Vinagre B.M., Podlubny I., Hernandez H., Feliu V., 2000, Some approximations of fractional order operators used in control theory and applications, Fractional Calculus and Applied Analysis, 3, 3, 231-248

16. Xue D., Chen Y.Q., Atherton D., 2001, Linear feedback control - analysis and design with Matlab 6.5, Advances in Design and Control 